RESCEU-7/14

\title{
Reheating the Universe Once More: The Dissipation of Acoustic Waves as a Novel Probe of Primordial Inhomogeneities on Even Smaller Scales
}

\author{
Tomohiro Nakama ${ }^{1,2}$, Teruaki Suyama ${ }^{2}$, and Jun'ichi Yokoyama ${ }^{2,3}$ \\ 1 Department of Physics, Graduate School of Science, \\ The University of Tokyo, Tokyo 113-0033, Japan \\ 2 Research Center for the Early Universe (RESCEU), Graduate School of Science, \\ The University of Tokyo, Tokyo 113-0033, Japan and \\ ${ }^{3}$ Kavli Institute for the Physics and Mathematics of the Universe (Kavli IPMU), WPI, TODIAS, \\ The University of Tokyo, Kashiwa, Chiba 27\%-8568, Japan
}

(Dated: September 11, 2021)

\begin{abstract}
We provide a simple but robust bound on the primordial curvature perturbation in the range $10^{4} \mathrm{Mpc}^{-1}<k<10^{5} \mathrm{Mpc}^{-1}$, which has not been constrained so far unlike low wavenumber modes. Perturbations on these scales dissipate the energy of their acoustic oscillations by the Silk damping after primordial nucleosynthesis but before the redshift $z \sim 2 \times 10^{6}$ and reheat the photon bath without invoking CMB distortions. This acoustic reheating results in the decrease of the baryonphoton ratio. By combining independent measurements probing the nucleosynthesis era and around the recombination epoch, we find an upper bound on the amplitude of the curvature perturbation over the above wavenumber range as $\mathcal{P}_{\zeta}<0.06$. Implications for super massive black holes are also discussed.
\end{abstract}

PACS numbers:

Introduction. Primordial inhomogeneities have been intensively investigated by cosmic microwave background $(\mathrm{CMB})$ 1, 2] or large scale structures of the Universe. However, the perturbation scales relevant to these probes are limited to $\mathcal{O}(\mathrm{Mpc})$ to $\mathcal{O}(\mathrm{Gpc})$ and information of fluctuations on smaller scales is relatively scarce. On the other hand, some models of the early universe predict enhancement of the power spectrum of fluctuations on small scales [3 16], so investigating small scale perturbations is important. Given this situation, several methods to probe small scale fluctuations have been studied such as primordial black holes (PBHs) [17, 18], ultracompact minihalos 19 23], and CMB spectral distortions 24 33].

In this Letter, we propose a novel method to probe perturbations on smaller scales than those probed by $\mathrm{CMB}$ spectral distortions $\left(10^{4} \mathrm{Mpc}^{-1}<k\right)$. This method is based on a phenomenon we call "acoustic reheating". During the radiation-dominated era, perturbations are damped after the horizon crossing (diffusion damping or Silk damping [34, 35]), injecting energy into the background universe. Before the $\mu$-era, or the epoch when energy release leads to $\mu$-distortions, any energy injection only causes increase in the average photon temperature, without invoking any spectral distortions 36 38]. If this energy injection takes place after the Big Bang Nucleosynthesis (BBN), it increases the number density of photons $n_{\gamma}$, without changing the number density of baryons $n_{\mathrm{b}}$, and so decreases the baryon-photon ratio $\eta \equiv n_{\mathrm{b}} / n_{\gamma}$. Since the value of $\eta$ is independently inferred by BBN 39] and CMB observation [40], we can put constraints on the amount of energy injection [41], or primordial perturbation amplitude (see also [42]).

Calculation of energy injection. The basic equations can be found in [26] (hereafter CEB). The total energy release due to the damping of acoustic waves from the redshift $z_{2}$ to $z_{1}\left(<z_{2}\right)$ is given by

$$
\frac{\Delta \rho_{\gamma}}{\rho_{\gamma}}=\int_{z_{1}}^{z_{2}} \frac{1}{a^{4} \rho_{\gamma}} \frac{d\left(a^{4} Q_{\mathrm{ac}}\right)}{d z} d z
$$

with

$$
\frac{1}{a^{4} \rho_{\gamma}} \frac{d\left(a^{4} Q_{\mathrm{ac}}\right)}{d z} \sim 9.4 a \int \frac{k d k}{k_{\mathrm{D}}^{2}} \mathcal{P}_{\zeta}(k) 2 \sin ^{2}\left(k r_{\mathrm{s}}\right) e^{-2 k^{2} / k_{\mathrm{D}}^{2}},
$$

where $r_{\mathrm{s}} \sim 2.7 \times 10^{5}(1+z)^{-1} \mathrm{Mpc}$ is the sound horizon and $k_{\mathrm{D}} \sim 4.0 \times 10^{-6}(1+z)^{3 / 2} \mathrm{Mpc}^{-1}$ is the damping scale determined by the diffusion of photons.

The largest contributions to the energy release at a redshift $z$ come from the modes around $k \sim k_{\mathrm{D}}(z)$ and so we can safely approximate $\sin ^{2}\left(k r_{\mathrm{s}}\right) \sim 1 / 2$, since $k_{\mathrm{D}}(z) r_{\mathrm{s}}(z) \sim(1+z)^{1 / 2} \gg 1$. Let us consider a top-hat power spectrum: $\mathcal{P}_{\zeta}(k)=A_{\zeta}\left(k_{1}<k<k_{2}\right), 0$ (otherwise), noting that the effects of acoustic reheating are most significant when the width of the enhanced part of the power spectrum is widest. We set $k_{1}=10^{4} \mathrm{Mpc}^{-1}$, since the power spectrum is severely constrained for $k_{1}<10^{4} \mathrm{Mpc}^{-1}$ by $\mu$-distortion [26]. On the other hand, the modes $10^{5} \mathrm{Mpc}^{-1}<k$ dissipate before the neutrino decoupling due to the neutrino diffusion. The comoving wave number for the neutrino diffusion becomes $k=10^{5} \mathrm{Mpc}^{-1}$ at the time of neutrino decoupling, which is close to the horizon scale at that time 43]. Since what can be probed by the consistency between BBN and CMB is only energy injection after BBN, taking place shortly after the neutrino decoupling, modes shorter than $k=10^{5} \mathrm{Mpc}^{-1}$ cannot be constrained and so we set $k_{2}=10^{5} \mathrm{Mpc}^{-1}$. Correspondingly, we choose $z_{1}=2 \times 10^{6}$, the onset of $\mu$-era, and $z_{2}=8.5 \times 10^{6}$, 
around when the mode $k=10^{5} \mathrm{Mpc}^{-1}$ dissipates, assuming it dissipates due to the diffusion of photons.

For the top-hat power spectrum ranging from $k_{1}$ to $k_{2}$, the energy release given by Eq. (2) becomes

$$
\begin{array}{r}
\frac{1}{a^{4} \rho_{\gamma}} \frac{d\left(a^{4} Q_{\mathrm{ac}}\right)}{d z} \sim \frac{2.4 A_{\zeta}}{1+z}\left[\exp \left\{-2\left(\frac{1+z_{*, 1}}{1+z}\right)^{3}\right\}\right. \\
\left.-\exp \left\{-2\left(\frac{1+z_{*, 2}}{1+z}\right)^{3}\right\}\right]
\end{array}
$$

where $k_{*} \equiv 4 \times 10^{-6} \mathrm{Mpc}^{-1}$ and

$$
z_{*, i} \equiv\left(\frac{k_{i}}{k_{*}}\right)^{2 / 3}(i=1,2)
$$

is the redshift when the mode $k_{i}$ dissipates. The total energy release then becomes

$$
\begin{aligned}
& \frac{\Delta \rho_{\gamma}}{\rho_{\gamma}} \sim 0.8 A_{\zeta}\left[\operatorname{Ei}\left(-2\left(\frac{1+z_{*, 1}}{1+z}\right)^{3}\right)\right. \\
& \left.-\operatorname{Ei}\left(-2\left(\frac{1+z_{*, 2}}{1+z}\right)^{3}\right)\right]_{z_{2}}^{z_{1}} \sim 2.3 A_{\zeta},
\end{aligned}
$$

where Ei denotes an exponential integral.

Constraints on $A_{\zeta}$ obtained by the baryon-photon ratio. The baryon-photon ratio $\eta$ has been determined independently by BBN and CMB, so the damping should not increase the number of photons too much (or equivalently should not decrease $\eta$ too much) after BBN, from which constraints on $A_{\zeta}$ can be obtained. To be consistent with observation, we require (noting $n_{\gamma} \propto T^{3}, \rho_{\gamma} \propto T^{4}$ )

$$
\frac{\eta_{\mathrm{CMB}}}{\eta_{\mathrm{BBN}}}=\left(1-\frac{3}{4} \frac{\Delta \rho_{\gamma}}{\rho_{\gamma}}\right)>\frac{\eta_{\mathrm{CMB}, \mathrm{obs}}}{\eta_{\mathrm{BBN}, \mathrm{obs}}},
$$

where $\eta_{\mathrm{BBN}}$ and $\eta_{\mathrm{CMB}}$ are the baryon-photon ratios at the time of BBN and after the onset of the $\mu$-era ( $\eta$ becomes constant after this moment since we only consider energy injection before the $\mu$-era); the subscript "obs" implies a value determined by observation. Using (5), this inequality is rewritten as a constraint on $A_{\zeta}$ :

$$
A_{\zeta} \lesssim 0.6\left(1-\frac{\eta_{\mathrm{CMB}, \mathrm{obs}}}{\eta_{\mathrm{BBN}, \mathrm{obs}}}\right) .
$$

As the observed values, we follow 39], in which $\eta=$ $(6.11 \pm 0.08) \times 10^{-10}$ for $\mathrm{CMB}$ and $\eta=(6.19 \pm 0.21) \times$ $10^{-10}$ for BBN were adopted. For $1 \sigma$ constraint, we conservatively set $\eta_{\mathrm{CMB}, \mathrm{obs}}=(6.11-0.08) \times 10^{-10}$ and $\eta_{\mathrm{BBN}, \mathrm{obs}}=(6.19+0.21) \times 10^{-10}$ (and $2 \sigma$ constraints are considered similarly). Then, the constraint on $A_{\zeta}$ is

$$
A_{\zeta} \lesssim 0.03(1 \sigma), \quad 0.06(2 \sigma) .
$$

Discussion. The constraints on the amplitude of the curvature perturbation have also been obtained to avoid overproduction of PBHs to be consistent with observations [44 46]. If we follow [18] (see also 17]), considering the disruption of wide binaries, which is relevant to the scales accessible by acoustic reheating, we obtain a constraint by $\mathrm{PBHs}$ as $A_{\zeta} \lesssim 0.05$.

Although the order of magnitude of these constraints is the same, we may not compare the two directly for several reasons. First a constraint imposed by PBH refers to the average of peaked curvature fluctuations over one e-fold of wavenumber, and it is obtained under the assumption that there is one-to-one correspondence between the mass of PBHs and the comoving scale of perturbation. But this is not true since the critical phenomenon [47, 48] has been observed, which results in formation of a number of $\mathrm{PBHs}$ with their mass much smaller than the comoving horizon mass. Furthermore, since PBHs are created at high$\sigma$ peaks, possible non-Gaussian distribution may change their abundance in a model dependent manner [3, 5, 11]. In particular, when non-Gaussianity is extremely large, it can change constraints to avoid overproduction of $\mathrm{PBHs}$ [49, 50]. (Note that $f_{N L}^{\text {local }}=\mathcal{O}(1)$ corresponds to a case with extremely large non-Gaussianity for $\mathrm{PBH}$ formation whose relevant amplitude of fluctuation is $\mathcal{O}(0.1)$ and the ratio of linear-to- second-order term is as large as 0.1 for $f_{N L}^{\text {local }}=\mathcal{O}(1)$.)

On the other hand, acoustic reheating considered here is insensitive to the assumption of Gaussianity (as is also pointed out in CEB) and is relatively easy to quantify precisely as well as relate to observations. Furthermore, what is interesting about constraints on the amplitude of primordial fluctuations obtained by acoustic reheating is that they can improve almost in proportion to potential future decrease in error bars associated with the determination of $\eta$.

Though our constraints apply only in a relatively narrow range $10^{4} \mathrm{Mpc}^{-1}<k<10^{5} \mathrm{Mpc}^{-1}$, this technique will have profound implications. For example, if the constraints from acoustic reheating become tighter in future, PBHs in the corresponding comoving horizon mass range $10^{3} M_{\odot}<M<10^{5} M_{\odot}$ will be severely constrained (note that PBHs bigger than $10^{5} M_{\odot}$ are severely constrained by $\mu$-distortion [51]). This mass range is particularly interesting in the context of scenarios of PBHs as the seeds of super massive black holes.

Acknowledgements. This work was partially supported by JSPS Grant-in-Aid for Scientific Research 23340058 (J.Y.), Grant-in-Aid for Scientific Research on Innovative Areas No. 24103006 (J.Y.) and No. 25103505 (T.S.), and Grant-in-Aid for JSPS Fellow No. 25.8199 (T.N.).

Note added. During the final stages of preparing the manuscript we became aware of work by Jeong, Pradler, Chluba and Kamionkowski, who also observed the importance of reheating from second-order perturbations focusing, however, on different aspects of BBN constraints and obtaining different results [52]. We are grateful to the authors of [52] for useful communications in revising our manuscript. 
[1] G. Hinshaw et al. (WMAP Collaboration) (2012), 1212.5226 .

[2] P. Ade et al. (Planck Collaboration) (2013), 1303.5082.

[3] J. Garcia-Bellido, A. Linde, and D. Wands, Phys. Rev. D 54, 6040 (1996).

[4] H. M. Hodges and G. R. Blumenthal, Phys. Rev. D 42, 3329 (1990).

[5] P. Ivanov, P. Naselsky, and I. Novikov, Phys. Rev. D 50, 7173 (1994).

[6] J. Yokoyama, Astron. Astrophys. 318:673 (1997).

[7] J. Yokoyama, Phys. Rev. D 58, 083510 (1998).

[8] J. Yokoyama, Phys. Rep. 307, 133 (1998).

[9] M. Kawasaki and T. Yanagida, Phys. Rev. D 59, 043512 (1999).

[10] J. Yokoyama, Prog. Theor. Phys. Suppl. 136, 338 (1999).

[11] R. Saito, J. Yokoyama, and R. Nagata, J. Cosmol. Astropart. Phys. 2008, 024 (2008).

[12] A. Taruya, Phys. Rev. D 59, 103505 (1999).

[13] B. A. Bassett and S. Tsujikawa, Phys. Rev. D 63, 123503 (2001).

[14] A. M. Green and K. A. Malik, Phys. Rev. D 64, 021301 (2001).

[15] M. Kawasaki, T. Takayama, M. Yamaguchi, and J. Yokoyama, Mod. Phys. Lett. A22, 1911 (2007).

[16] T. Kawaguchi, M. Kawasaki, T. Takayama, M. Yamaguchi, and J. Yokoyama, Mon. Not. Roy. Astron. Soc. 388, 1426 (2008), 0711.3886.

[17] E. Bugaev and P. Klimai, Phys.Rev. D79, 103511 (2009), 0812.4247.

[18] A. S. Josan, A. M. Green, and K. A. Malik, Phys.Rev. D79, 103520 (2009), 0903.3184.

[19] T. Bringmann, P. Scott, and Y. Akrami, Phys.Rev. D85, 125027 (2012), 1110.2484.

[20] A. S. Josan and A. M. Green, Phys.Rev. D82, 083527 (2010), 1006.4970.

[21] F. Li, A. L. Erickcek, and N. M. Law, Phys.Rev. D86, 043519 (2012), 1202.1284.

[22] P. Scott, T. Bringmann, and Y. Akrami, J.Phys.Conf.Ser. 375, 032012 (2012), 1205.1432.

[23] Y. Yang, G. Yang, and H. Zong, Phys.Rev. D87, 103525 (2013), 1305.4213.

[24] J. Barrow and P. Coles, Mon. Not. Roy. Astron. Soc. 248, 52 (1991).

[25] J. Chluba and R. Sunyaev (2011), 1109.6552.

[26] J. Chluba, A. L. Erickcek, and I. Ben-Dayan, Astrophys.J. 758, 76 (2012), 1203.2681.
[27] J. Chluba, R. Khatri, and R. A. Sunyaev (2012), 1202.0057.

[28] J. B. Dent, D. A. Easson, and H. Tashiro, Phys.Rev. D86, 023514 (2012), 1202.6066.

[29] R. Khatri and R. A. Sunyaev, JCAP 1209, 016 (2012), 1207.6654 .

[30] R. A. Sunyaev and R. Khatri, Int.J.Mod.Phys. D22, 1330014 (2013), 1302.6553.

[31] R. Khatri and R. A. Sunyaev, JCAP 1306, 026 (2013), 1303.7212

[32] J. Chluba and D. Grin, Mon.Not.Roy.Astron.Soc. 434, 1619 (2013), 1304.4596.

[33] J. Chluba and D. Jeong (2013), 1306.5751.

[34] J. Silk, Astrophys.J. 151, 459 (1968).

[35] H. Sato, Prog. Theor. Phys. 45, 370 (1970).

[36] R. Sunyaev and Y. Zeldovich, Astrophys.Space Sci. 7, 20 (1970).

[37] C. Burigana, L. Danese, and G. de Zotti, Astron. Astrophys. 246, 49 (1991).

[38] W. Hu and J. Silk, Phys.Rev. D48, 485 (1993).

[39] G. Steigman and K. M. Nollett (2014), 1401.5488.

[40] P. Ade et al. (Planck Collaboration) (2013), 1303.5076.

[41] V. Simha and G. Steigman, JCAP 0806, 016 (2008), 0803.3465

[42] J. Barrow, Nature 267, 117 (1977).

[43] K. Jedamzik, V. Katalinic, and A. V. Olinto, Phys.Rev. D57, 3264 (1998), astro-ph/9606080.

[44] R. Saito and J. Yokoyama, Phys. Rev. Lett. 102, 161101 (2009), [107, 069901(E) (2011).], 0812.4339.

[45] R. Saito and J. Yokoyama, Prog. Theor. Phys. 123, 867 (2010), [126, 351(E) (2011).], 0912.5317.

[46] B. Carr, K. Kohri, Y. Sendouda, and J. Yokoyama, Phys. Rev. D 81, 104019 (2010), 0912.5297.

[47] J. C. Niemeyer and K. Jedamzik, Phys. Rev. D 59, 124013 (1999).

[48] I. Musco, J. C. Miller, and A. G. Polnarev, Class. Quant. Grav. 26, 235001 (2009), 0811.1452.

[49] C. T. Byrnes, E. J. Copeland, and A. M. Green, Phys.Rev. D86, 043512 (2012), 1206.4188.

[50] S. Young and C. T. Byrnes, JCAP 1308, 052 (2013), 1307.4995.

[51] K. Kohri, T. Suyama, and T. Nakama, in prep. (2014).

[52] D. Jeong, J. Pradler, J. Chluba, and M. Kamionkowski (2014), 1403.3697. 